\title{
Estrategias didácticas en el nivel universitario desde un enfoque dinámico de aprendizaje
}

\section{Didactic strategies at the university level from a dynamic learning approach}

\author{
Rita Azucena Maridueña Torres ${ }^{1}$ \\ rita englishteacher@hotmail.com \\ ORCID: https://orcid.org/0000-0002-7722-8527
}

Recibido: 28/9/2021, Aceptado: 28/12/2021

\begin{abstract}
RESUMEN
En Ecuador, el sistema innovador de estudios universitarios ha incorporado los métodos apropiados de enseñanza como parte fundamental del quehacer pedagógico con la finalidad de adecuar y ajustar los diferentes patrones metodológicos en el desarrollo de competencias y que a la vez permiten fortalecer la capacidad de respuestas de los estudiantes ante los constantes cambios en el ámbito formativo. La presente investigación tiene como propósito analizar la conceptualización de las estrategias didácticas en el nivel universitario desde un enfoque dinámico de aprendizaje a través de la aplicación de actividades como: clases magistrales, talleres, foros de discusión y proyectos las cuales facilitan la construcción continua del conocimiento y habilidades. Cabe destacar que cada una de estas acciones presentan sus propias características colaborativas, resolutivas, analíticas y comunicativas. Y todas estas actividades se deben aplicar mediante el LMS (Sistema de Gestión de Aprendizaje). Para realizar este análisis, se consideró los métodos de investigación descriptiva y explicativa. Además, se empleó las experiencias referenciadas en el entorno educativo para el uso de información en relevancia a la metodología centrada en el estudiante que implica un enfoque dinámico de aprendizaje. Los resultados expuestos fueron recomendables y favorables para la comunidad universitaria considerando la efectividad de las diferentes actividades aplicadas en todos los niveles para lograr un mejor rendimiento académico.
\end{abstract}

Palabras clave: Estrategias didácticas, nivel universitario, actividades formativas

\section{ABSTRACT}

In Ecuador, the innovative system of university studies has incorporated the appropriate teaching methods as a fundamental part of the pedagogical task in 


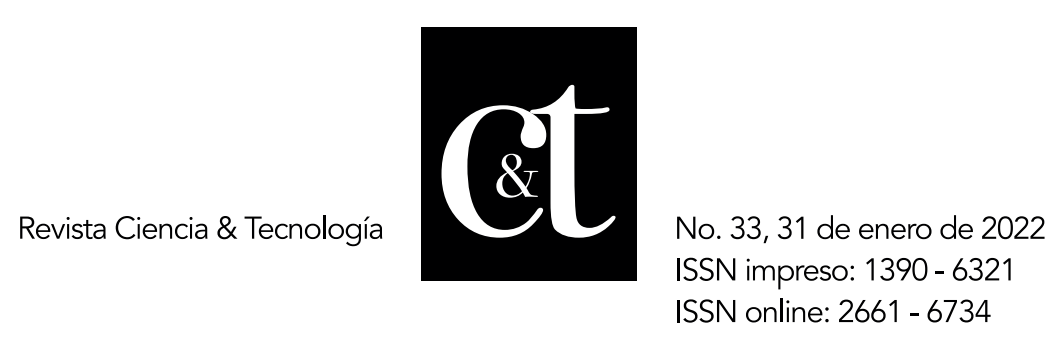

order to adapt and adjust the different methodological patterns in the development of competencies and that at the same time allow to strengthen the response capacity of students in the face of constant changes in the educational field. The purpose of this research is to analyze the conceptualization of didactic strategies at the university level from a dynamic learning approach through the application of activities such as: master classes, workshops, discussion forums and projects which facilitate the continuous construction of knowledge and skills. It should be noted that each of these actions has its own collaborative, decisive, analytical and communicative characteristics. And all these activities must be applied through the LMS (Learning Management System). To carry out this analysis, descriptive and explanatory research methods were considered. In addition, the experiences referenced in the educational environment were used for the use of information relevant to the student-centered methodology that implies a dynamic approach to learning. The results presented were recommended and favorable for the university community considering the effectiveness of the different activities applied at all levels to achieve better academic performance.

Keywords: Didactic strategies, university level, formative activities

\author{
${ }^{1}$ Magíster en Educación Superior \\ Diploma Superior en Docencia Universitaria \\ Docente de la Universidad Tecnológica Empresarial de Guayaquil, Guayaquil - Ecuador

\section{Introducción}

En Ecuador, el sistema innovador de estudios universitarios ha incorporado los métodos apropiados de enseñanza como parte fundamental del quehacer pedagógico con la finalidad de adecuar y ajustar los diferentes patrones metodológicos en el desarrollo de competencias y que a la vez permiten fortalecer la capacidad de respuestas de los estudiantes ante los constantes cambios en el ámbito formativo.

La presente investigación tiene como propósito analizar la conceptualización de las estrategias didácticas en el nivel universitario desde un enfoque dinámico de aprendizaje a través de la aplicación de actividades como: clases magistrales, talleres, foros de discusión y proyectos, las cuales facilitan la construcción continua del conocimiento y habilidades. Considerando que es necesario desarrollar estas actividades mediante uno de los métodos de enseñanza de mayor funcionalidad en el quehacer educativo, el método de Aprendizaje Basado en Proyectos, como una metodología centrada en el estudiante que implica un enfoque dinámico de aprendizaje. Así mismo, desde el punto de vista de (Muñoz, 2017) quien define al Aprendizaje Basado en Proyectos (ABP) como "una modalidad de enseñanza y aprendizaje centrada en tareas, un proceso compartido de negociación entre los participantes, siendo su objetivo principal la obtención de un producto final" (pág. 113). Se puede destacar la dedicación y esmero de los estudiantes en realizar dichas actividades.

De ahí que, se debe tener en cuenta su implementación dentro del proceso de enseñanza puesto que constituye la apropiación, integración y construcción de los conocimientos que el estudiante desea adquirir con la finalidad de generar habilidades competitivas. 


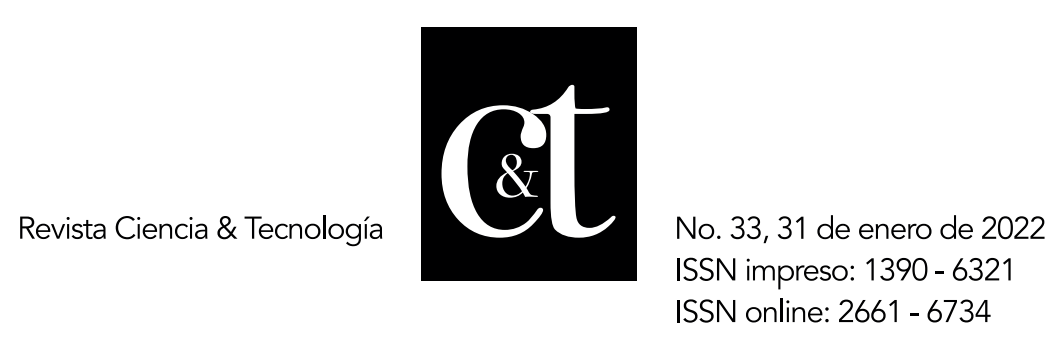

Por ello, este método actual de enseñanza se lo considera como una estrategia o técnica didáctica integral para lograr la formación y evaluación de las competencias. Cabe destacar que cada una de estas acciones formativas presentan sus propias características colaborativas, resolutivas, analíticas y comunicativas. Es decir, que dentro de estos entornos de aprendizaje dinámicos están los trabajos colaborativos, la resolución de problemas, el desarrollo del pensamiento crítico y la comunicación efectiva. Todos ellos deben estar relacionados al tema y contenido a estudiar.

Por otro lado, es preciso mencionar que todas estas actividades se deben aplicar mediante el LMS (Sistema de Gestión de Aprendizaje) con las adecuadas y coherentes orientaciones didácticas en el proceso formativo. $Y$ es por esto que es preciso plantear el siguiente interrogante: ¿Se consideran las estrategias didácticas como una contribución pedagógica - innovadora en el nivel universitario?

\section{Estrategias didácticas}

Por lo que se refiere a las estrategias didácticas es necesario indicar que existe un grado de comparación con lo que respecta a la aplicación de las técnicas y recursos en el salón de clase con los procedimientos de enseñanza en el quehacer de los docentes. Siendo más específicos se puede decir que las estrategias son las técnicas o recursos utilizados para canalizar el contenido a estudiar mientras que al hablar de procedimientos, nos referimos a las formas o manera como transmitimos el contenido que los estudiantes deben aprender.

En todas las épocas, las y los docentes se han preocupado por manejar formas y procesos que les ayuden a que sus alumnas y alumnos aprendan más y mejor. La didáctica es la disciplina pedagógica que responde a esta inquietud, pues centra su atención en la instrucción o formación intelectual, con sus métodos, técnicas y estrategias (Guzmán, 2001, pág. 25).

De esta manera se puede expresar que el propósito que persigue la aplicación de estas estrategias didácticas en el ámbito escolar es transmitir información o conocimientos, así como para las enseñanzas consideradas como más arduas o complejas dadas en las diferentes disciplinas y poder lograr optimizar la adquisición de los conocimientos, favoreciendo el aprendizaje de los estudiantes de aquellas habilidades o competencias que se hayan preestablecido como importantes.

Asimismo, cabe señalar los diferentes aportes de varios autores sobre la noción de estrategias didácticas. Según Díaz (2010) las define como: "procedimientos y recursos que utiliza el docente para promover aprendizajes significativos, facilitando intencionalmente un procesamiento del contenido nuevo de manera más profunda y consciente" ( $p$. 118). Teniendo en cuenta que para Tobón (2010) las estrategias didácticas son "un conjunto de acciones que se proyectan y se ponen en marcha de forma ordenada para alcanzar un determinado propósito", por ello, en el campo pedagógico específica que se trata de un "plan de acción que pone en marcha el docente para lograr los aprendizajes" (p. 246). 


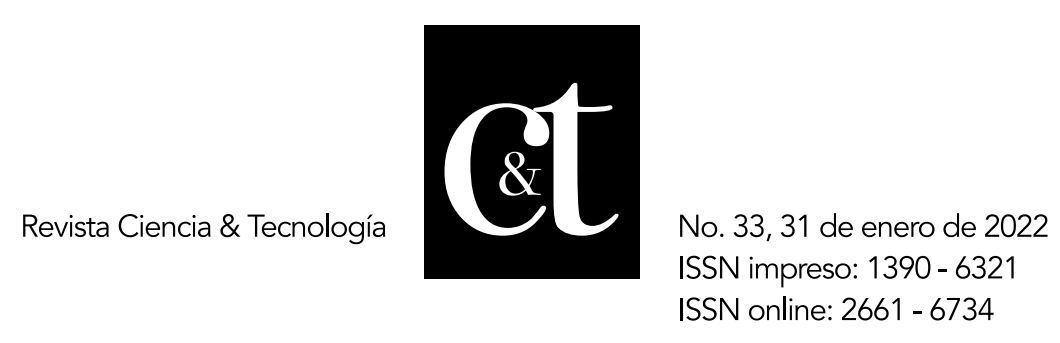

Empleando las palabras de Colom, Salinas y Sureda (1988) utilizaron el concepto de estrategia didáctica como una instancia que acoge tanto métodos, como medios y técnicas, considerando que el concepto proporcionaba mayor flexibilidad y utilidad en el proceso didáctico. En tal sentido, las estrategias didácticas son consideradas como recursos pedagógicos empleadas en el campo educativo ya que deben ser aplicadas en las diferentes disciplinas para poder transmitir aquellos saberes que están siendo impartidos.

Por otra parte, Rovira (2021) da a conocer que una de las principales características de las estrategias didácticas es que estas requieren de una planificación y organización previa. Para que estas estrategias puedan ser aplicadas dentro del aula, el docente deberá tener en cuenta los siguientes aspectos:

- Establecer los objetivos específicos a conseguir dentro de una materia, disciplina o aprendizaje concreto.

- Poseer los conocimientos necesarios para la trasmisión de la información.

- Prever y preparar todos aquellos materiales u objetos que serán necesarios para la enseñanza.

- Enfatizar los aspectos importantes de la información que se quiere transmitir.

- Promover la asociación de los conocimientos teóricos con los aspectos prácticos de estos.

- Fomentar la autonomía del alumno a la hora de generar estrategias propias de aprendizaje.

Conviene subrayar que la planificación y la organización de las estrategias didácticas son de suma importancia ya que el docente tutor debe facilitar el aprendizaje y a la vez ser una orientación en la adquisición de aprendizaje. Ahora, si bien es cierto dentro de la elaboración de la planificación de dichas estrategias didácticas se deben crear los objetivos específicos acordes al contenido de la asignatura a estudiar y dominar el tema a impartir.

Así mismo, buscar la relación entre los conocimientos teóricos y prácticos con la finalidad de obtener un resultado favorable en el proceso de aprendizaje. Además, se trata de incrementar la independencia de cada estudiante en el momento de crear las estrategias propias de aprendizaje.

En cuanto a la utilización de las estrategias didácticas actualmente en las aulas universitarias, permiten generar la interacción entre el profesor y el grupo de estudiantes y trabajar unidos en la construcción del aprendizaje logrando que los estudiantes adquieran un papel activo, desarrollando un sentido de responsabilidad frente a su aprendizaje. Para ello, es recomendable implementar las siguientes estrategias didácticas dentro del proceso innovador de aprendizaje:

\section{Clases magistrales}

Dentro del proceso de enseñanza y aprendizaje, Pujol, J y Fons, J.L (1989) afirman que las clases magistrales son consideradas "Como método de enseñanza, nació con la misma universidad...," y que por su condición 
netamente pedagógica ha recibido muchas críticas y por su finalidad de transmitir conocimientos seleccionados a un grupo masivo de estudiantes puesto que solo ellos son dedicados a escuchar, tomar notas. En otras palabras, la actividad principal está en la acción que involucra y desarrolla el docente tutor, quien se destaca en cumplir con su rol de facilitador e instructor para que los estudiantes entiendan un tópico determinado a través de conceptos mencionados.

En efecto, la calidad y efectividad de una clase magistral responde a criterios de orden didáctico, adaptación a los estudiantes, organización de los contenidos, presentación de esquemas, secuenciar la exposición, elegir los medios para exponer, tener en cuenta los ritmos de atención y la retroalimentación de los estudiantes considerando esta nueva modalidad innovadora de enseñanza ya que se puede incorporar una serie de herramientas pedagógicas con el fin de que la clase sea más ilustrativa, participativa, interactiva y comunicativa y que a la vez faciliten una clase dinámica en el momento de la adquisición de los nuevos conocimientos.

\section{Talleres}

Hoy en día dentro del ámbito educativo se ha considerado al taller como estrategia pedagógica siendo una de las herramientas pedagógicas propuestas con mayor acierto formativo. Según Vasco, un taller "...es un ambiente educativo en el cual la interacción con el conocimiento es también interactiva e intersubjetiva entre los participantes, de manera que genere procesos individuales en cada uno de los participantes..." $\mathrm{y}^{\prime \prime}$...hacer un taller es vivir una experiencia, propone un ambiente rico en recursos, de manera que genere procesos individuales y grupales que permitan socializar los procesos personales de cada uno de los participantes".

Es preciso mencionar los aspectos que se deben considerar en el momento de diseñar el esquema de un taller con propósitos académicos. Estos son: tema de la actividad, objetivo, descripción, orientaciones tanto metodológicas como prácticas y los respectivos lineamientos generales. Entonces, cabe recalcar que el desarrollo de cada taller como actividad de cierre de un bloque didáctico permite promover la interdisciplinariedad, la integración de la práctica y la teoría, el trabajo en equipo y el apoyo al educando a aprender a aprender.

Al mismo tiempo, los estudiantes logran trabajar en resolución de problemas, contrastación de hipótesis, debates, comparación de ideas, interactividad e interacción y en situaciones de experimentación. Dicho de otra manera, el estudiante se convierte en el eje fundamental del proceso de enseñanzaaprendizaje: siendo así, éste se centra en el sujeto que aprende y no en el sujeto que enseña.

\section{Foros de discusión}

En el sistema educativo de modalidad online brinda otra de las actividades académicas con mayor disposición formativa, esta se refiere al foro de discusión puesto que se lo considera como un espacio de encuentro entre diversos 


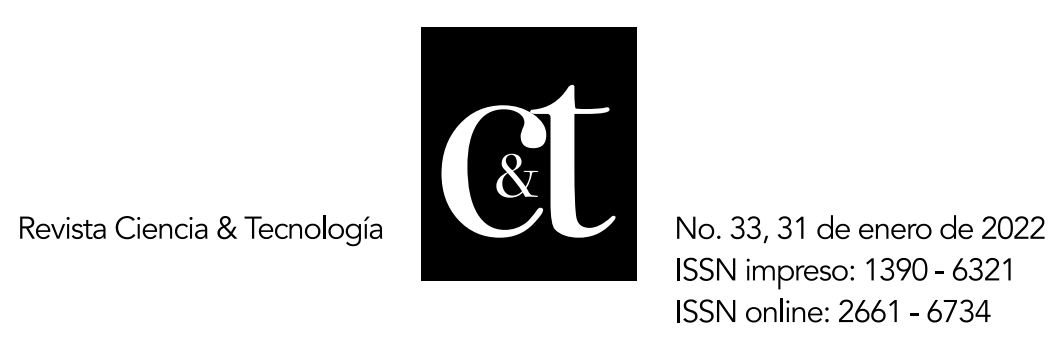

participantes, con la finalidad de intercambiar opiniones sobre un tema de interés común. Por lo general, la organización de un foro de discusión de naturaleza virtual se debe incluir los espacios habilitados para discusión en las respectivas plataformas.

Jonassen (1998), citado por Markel (2001), señala que "el foro virtual no es otro tipo de pizarra clásica o método de comunicación unidireccional, sino que constituye una herramienta tecnológica que, a través de una metodología apropiada, propicia la creación de los ambientes que estimulan el aprendizaje y el pensamiento crítico a través de la negociación y construcción del conocimiento". Empleando las palabras de (Domínguez y Alonso, 2004), "el foro virtual puede llegar a desempeñar el rol central en el modelo de la enseñanza aprendizaje orientado al desarrollo del pensamiento crítico". Otros expertos manifiestan que los foros virtuales pueden tener distintos enfoques y su valor educativo varía dependiendo de la forma de diálogo que prevalece.

Desde el punto de vista de Wilkins (2002) citado por Fëdorov (2006) plantea una vasta lista de autores contemporáneos donde recopila y amplía una serie de ventajas y desventajas del uso del foro virtual. Entre las ventajas se encuentran:

- Es flexible. Los estudiantes pueden leer y colocar sus aportes independientemente del horario presencial del curso.

- Permite que los alumnos formulen los pensamientos más profundos. Esto ocurre en gran medida debido a que ellos tienen todo el diálogo en forma escrita y suficiente tiempo para pensar. Además, les ayuda a mejorar sus habilidades de comunicación escrita.

- Facilita la participación y se adapta para las personas introvertidas, quienes no suelen participar en la discusión tradicional en clase.

- Agiliza el aprendizaje a través de la solución de problemas en forma grupal. Hay evidencia de que esto conduce a mayor desarrollo cognoscitivo, comparado con el trabajo individual.

- Permite generar un registro o trascripción completa de la discusión, lo que da a los usuarios la oportunidad de manipular, almacenar, recuperar, imprimir y corregir.

Entre las otras desventajas del uso de los tableros de discusión, mencionadas por Beth Wilkins (2002) se encuentran las siguientes:

- Los participantes pueden sentirse aislados cuando no reciben una respuesta inmediata a sus ideas y comentarios.

- Hay una pérdida de comunicación no verbal: como, por ejemplo, las expresiones, la voz, la entonación, los gestos faciales, etc.

- Es más difícil saber cuándo se ha agotada una conversación y es apropiado moverse a un nuevo tema.

- Inicialmente, el seguimiento y animación del foro requiere de mucho tiempo del profesor o del asistente.

- Es fácil llegar a tener una sobrecarga de información, ya que los estudiantes deben leer la discusión en línea además del material de la lectura del curso. 
De manera que es importante el compromiso del docente como tutor guiar y direccionar a los estudiantes con las respectivas instrucciones metodológicas al enviar cada una de las tareas a desarrollar en el foro de discusión. Así, por ejemplo, cuando los alumnos solo tienen que participar contestando un interrogante o dejando su opinión en un espacio virtual de discusión preestablecido.

\section{Proyectos}

En el ámbito pedagógico universitario se conoce que está representado por esta nueva técnica didáctica de adquirir los conocimientos y competencias generales en las diferentes disciplinas. Por ello, es necesario considerar la aplicación de esta estrategia de aprendizaje ya que permite ayudar facilitar un aprendizaje constructivo que supondrá una adquisición más significativa de conocimientos en los alumnos participantes. Como lo hace notar Navarro y otros autores (2011) señalando que:

El aprendizaje basado en proyectos es un modelo de enseñanza que involucra a los estudiantes en la indagación para resolver problemas interesantes, que presenta por finalidad un producto real. En los proyectos de aprendizaje, el conocimiento es construido de forma colectiva entre alumnos y profesores. Además, el aprendizaje basado en proyectos implica formar equipos integrados por alumnos con perfiles diferentes, lo que va en la línea de investigación que, nuestro proyecto ha seguido durante los dos últimos años, a cerca de los equipos de trabajo multidisciplinares y como preparar a los estudiantes para un contexto laboral y económico, diverso y global.

Por otro lado, es preciso mencionar a García y Pérez, quienes consideran que "entre las metodologías de aprendizaje activo el ABP tiene un lugar singular y describen un método para diseñar actividades docentes organizadas bajo esta estrategia, a partir del análisis de diferentes modelos, así como el diseño de una herramienta digital para su implementación". Puesto que existen una gran variedad de herramientas Web 2.0 para generar una comunicación efectiva entre los grupos de trabajo mediante una página Wiki, la cual permite modificar el contenido de las diferentes actividades grupales.

\section{Estrategias didácticas como una contribución pedagógica - innovadora en el proceso de aprendizaje en las aulas universitarias}

Dentro de los procesos dinámicos y sinérgicos se encuentran la educación, la enseñanza, el aprendizaje y el desarrollo, los cuales están asociados con las actividades formativas de los estudiantes, con el compromiso personal y responsabilidad y con las prácticas sociales. Aspectos que permiten avalar los avances científicos y tecnológicos, el contexto actual de la globalización y el auge dinámico de las tecnologías de la información y la comunicación con la finalidad de gestionar la información y el conocimiento, el sistema educativo en general y sus procesos específicos en particular.

En el campo educativo universitario es necesario mencionar que "El maestro tiene como función "facilitar y orientar el aprendizaje, guiando, asesorando y coordinando las actividades de los alumnos" (Arrendo, Pérez Rivera y Aguirre L. María, 1992, p. 17), considerando la característica propia del estudiante y su entorno, el conocimiento por compartir y enseñar, además de la organización, 


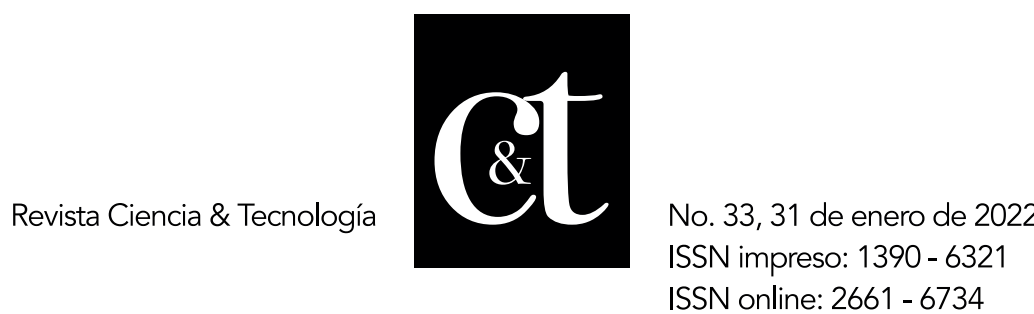

planificación, gestión, supervisión e innovación permanente de secuencias de aprendizaje y de recursos didácticos, estrategias y técnicas de enseñanza.

Ahora bien, es preciso considerar la estrategia de enseñanza como los procedimientos o recursos que consciente y planificadamente utiliza el maestro para promover los aprendizajes deseados. Si una estrategia es "la habilidad o destreza para dirigir un asunto", las estrategias de enseñanza se pueden considerar como aquellos modos de actuar del maestro que hacen que se generen aprendizajes, y por eso, estas estrategias son el producto de una actividad constructiva, creativa y experiencial del maestro, pensadas con anterioridad al ejercicio práctico de la enseñanza, dinámicas y flexibles según las circunstancias y momentos de acción. (Londoño y Calvache López ,2010, pág. 21).

Desde la posición de Campos (2000) en su libro Estrategias didácticas apoyadas en la tecnología señala que las estrategias de enseñanza se refieren a las operaciones o actividades utilizadas por el profesor para mediar, facilitar, promover, organizar aprendizajes y argumenta que la concepción, selección, producción y uso de recursos de aprendizaje están en relación con los demás componentes didácticos y las estrategias de enseñanza - aprendizaje (estrategias EA) y su función es mediar de diferentes formas el aprendizaje.

Así mismo, cabe resaltar que la renovación de las estrategias de enseñanza debe estar orientada a propiciar un aprendizaje autónomo de los estudiantes. La combinación de estrategias aparece como el camino más productivo para emprender la innovación de la docencia. Y en efecto, Las TIC constituyen una herramienta muy importante en este sentido puesto que ellas permiten asimilar los contenidos a estudiar de manera precisa, participativa y comunicativa con la gran ayuda de los recursos pedagógicos que existen en la web.

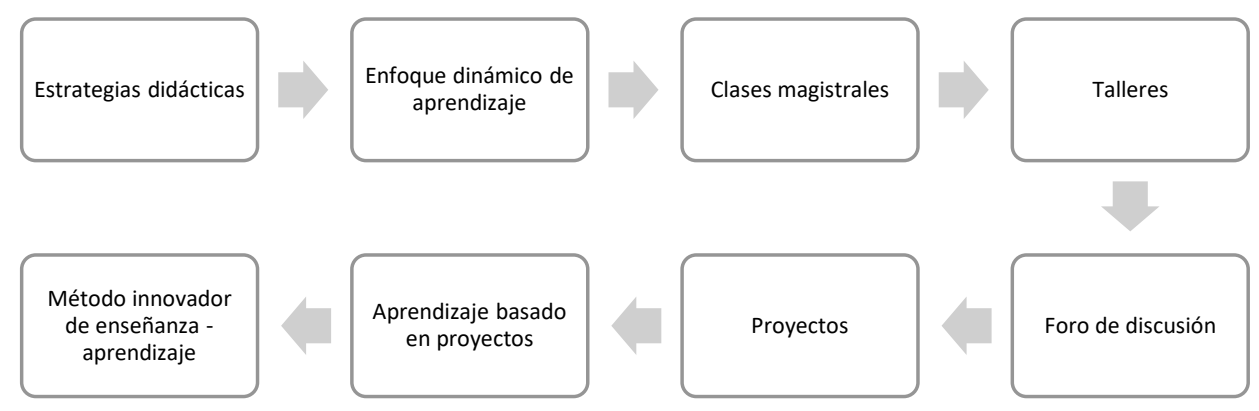

Gráfico 1 Contribución pedagógica - innovadora en el nivel universitario Fuente: Elaboración propia

De modo que las estrategias didácticas son realmente una contribución pedagógica - innovadora en el proceso de aprendizaje en las aulas universitarias porque ellas son técnica o recursos necesarios en el ámbito educativo que permiten fortalecer la adquisición de los aprendizajes y así lograr un resultado satisfactorio en el rendimiento académico. 


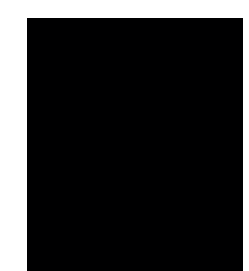

\section{Metodología}

Para realizar este análisis, se consideró los métodos de investigación descriptiva y explicativa de nivel interpretativo basado en una revisión documental bibliográfica alcanzando identificar factores como la caracterización, la interpretación y la aplicación dentro del proceso de enseñanza en las aulas universitarias. Además, se empleó las experiencias referenciadas en la comunidad educativa virtual para el uso de información en relevancia a la metodología centrada en el estudiante que implica un enfoque dinámico de aprendizaje.

\section{Resultados y discusión}

Los resultados mostrados fueron favorables para la comunidad universitaria considerando la efectividad de las diferentes actividades aplicadas en todos los niveles para lograr un mejor rendimiento académico. Con respecto a la obtención de los datos reflejados en el gráfico, se puede indicar que se consultó de manera online aleatoria a ciertos estudiantes y docentes de los diferentes niveles de estudio sobre la aplicación de estas actividades formativas en las aulas universitarias y señalaron que actualmente ellas son efectivas y pertinentes.

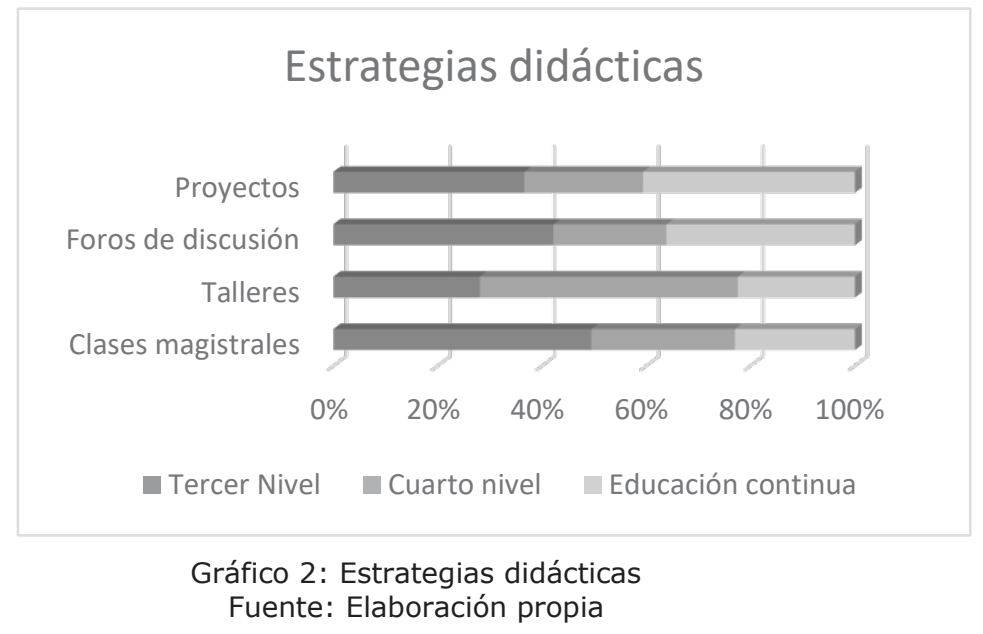

\section{Conclusiones}

En este trabajo se analizó la conceptualización y caracterización de las estrategias didácticas en el nivel universitario desde un enfoque dinámico de aprendizaje a través de la aplicación de procedimientos o técnicas formativas como: clases magistrales, talleres, foros de discusión y proyectos que facilitan la construcción continua del conocimiento y habilidades. El aspecto más importante del análisis de la conceptualización de las estrategias didácticas en el nivel universitario fue conocer más sobre el acto didáctico, el cual pretende lograr una "enseñanza activa" que involucra tanto la conducta del maestro en el ejercicio de la docencia como el compromiso y la responsabilidad del estudiante en el desempeño académico. 


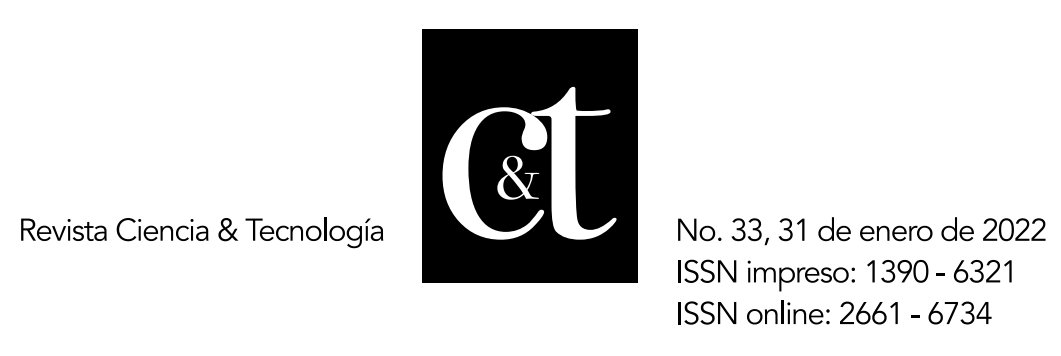

Es preciso también señalar que el aspecto que más ayudó a analizar esta conceptualización fue la consolidación de las experiencias académicas referenciadas para orientar a los docentes que buscan la transformación educativa a través de la implementación de estrategias innovadoras e integradoras de enseñanza logrando el propósito de aprender en los estudiantes, el contenido explícito a estudiar claro y especifico, el tiempo propicio para llevar a cabo las tareas asignadas, el aprendizaje explicativo y dinámico, y una enseñanza interactiva, participativa, activa y comunicativa porque estas son fundamentales para el desarrollo de la educación universitaria. Cabe resaltar que lo más difícil que se encontró en este análisis fue conocer los desafíos de las nuevas tecnologías que enfrentan los docentes universitarios actualmente.

En definitiva, el punto clave en el quehacer educativo será mantener el deseo de mejorar e innovar la labor de enseñanza para lograr el prestigio y la calidad de la docencia.

\section{Referencias}

Arteaga, I. H., Meneses, J. R., \& Luna, J. A. (2015). Estrategia didáctica: una competencia docente en la formación para el mundo laboral. Revista Latinoamericana de Estudios Educativos (Colombia), 11(1), 73-94.

Brito, V. (2004). El foro electrónico: una herramienta tecnológica para facilitar el aprendizaje colaborativo. Edutec. Revista electrónica de tecnología educativa, (17), a038-a038.

Campos, Y. (2000). Estrategias didácticas apoyadas en tecnología. México: Dgenamdf.

Cañizales, J. Y. (2004). Estrategias didácticas para activar el desarrollo de los procesos de pensamiento en el preescolar. Investigación y postgrado, 19(2), $179-200$.

Colom, A., Sureda, J., \& Salinas, J. (1988). Tecnología y medios educativos. Cincel.

De Pepa, M., \& Adriana, M. (2006). La utilización de foros virtuales en la universidad como metodología de aprendizaje colaborativo. Revista cognición, 8, 59-74.

Díaz Barriga Arceo, Frida y Hernández Rojas, Gerardo (2010). Estrategias Docentes para un aprendizaje significativo. Una interpretación constructiva. McGraw Hill. México; D.F.

Fëdorov, A. N. (2006). Foro virtual como una estrategia metodológica para el desarrollo del pensamiento crítico en la universidad. Innovación educativa, 6(30), 62-72. 


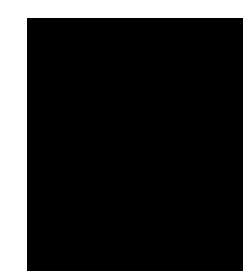

García; J. Pérez. Aprendizaje basado en proyectos: método para el diseño de actividades. Tecnología, Ciencia y Educación, (2018) 37-63. Recuperado el 11 de 04 de 2019, de

http://www.tecnologia-cienciaeducacion.com/index.php/TCE/article/view/194

Guzmán, A., \& Calderón, M. (2004). Orientaciones Didácticas para el Proceso Enseñanza-Aprendizaje. Ed. Amigo del Hogar. 2a. Edición. Santo Domingo.

Heladio. (2000). Tendencias educativas y pedagógicas. ABC de la evaluación docente. Bogotá: Ediciones SEM.

https://psicologiaymente.com/desarrollo/estrategias-didacticas

Luna, M. E. R. (2012). El taller: una estrategia para aprender, enseñar e investigar. Lenguaje y Educación: Perspectivas metodológicas y teóricas para su estudio, 13-43.

Martín, J. G., \& Martínez, J. E. P. (2018). Aprendizaje basado en proyectos: método para el diseño de actividades. Revista Tecnología, Ciencia y Educación, (10).

Monereo, Carlos y otros. (1998). Estrategias de enseñanza aprendizaje. México: SEP. Moreno M.,

Moreno M., Heladio. (2000). Biografía de grandes pedagogos. ABC de la evaluación. Bogotá: Editorial Géminis.

Muñoz-Repiso, A. G. V., \& Gómez-Pablos, V. B. (2017). Aprendizaje Basado en Proyectos (ABP): evaluación desde la perspectiva de alumnos de Educación Primaria. Revista de Investigación Educativa, 35(1), 113-131.

Navarro, I., Pertegal, M., Gil, D., González, C., \& Jimeno, A. (2011). El aprendizaje basado en proyectos como estrategia didáctica y pedagógica para estimular el desarrollo de competencias profesionales. IX Jornadas de redes de investigación en docencia universitaria.

Pazos, M., Pérez, A., \& Salinas, J. (2001, September). Comunidades virtuales: de las listas de discusión a las comunidades de aprendizaje. In Comunicación. Edutec'01. V Congreso Internacional de Tecnología, Educación y Desarrollo sostenible (pp. 17-19).

Penso, R. A. (2015). El taller como estrategia metodológica para estimular la investigación en el proceso de enseñanza-aprendizaje en la educación superior. Boletín Redipe, 4(10), 49-55.

Pilar Londoño Martínez, José Edmundo Calvache López. (2010) Las estrategias de enseñanza: aproximación teórico-conceptual.

Pujol, J y Fons, J.L (1989): Los Métodos en la Enseñanza Universitaria. Universidad de Navarra, Pamplona.

Robles Zepeda, F. J., \& Jiménez González, a. M. P. A. R. O. (2016). Las estrategias didácticas y su papel en el desarrollo del proceso de enseñanza aprendizaje. Conacyt. 


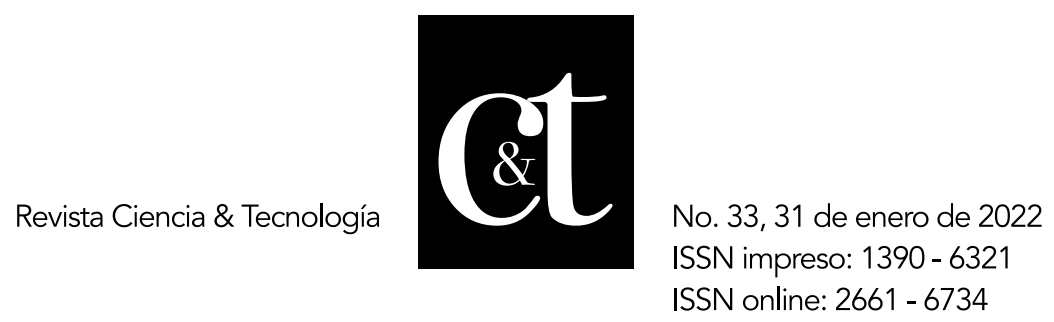

Rovira, I., (2021) Estrategias didácticas: definición, características y aplicación Sierra Gómez, H. (2013). Aprendizaje activo como mejora de las actitudes de los estudiantes hacia el aprendizaje.

Tobón, Tobón M (2010). Formación integral y competencia, Pensamiento Complejo, diseño curricular y didáctica. ECOE. Bogotá Colombia.

Travieso Valdés, D., \& Ortiz Cárdenas, T. (2018). Aprendizaje basado en problemas y enseñanza por proyectos: alternativas diferentes para enseñar. Revista Cubana de Educación Superior, 37(1), 124-133.

Vásquez, F. (2010). Estrategias de enseñanza: investigaciones sobre didáctica en instituciones educativas de la ciudad de Pasto.

Wilkins, E. (2002). Facilitación del aprendizaje en línea: Capacitación de asistentes técnicos para facilitar la comunidad, la colaboración y la tutoría en el entorno en línea (Tesis doctoral, Universidad Brigham Young. Departamento de Psicología y Tecnología de la Instrucción). 